\title{
Formation of B19', B2, and amorphous phases during mechano-synthesis of nanocrystalline NiTi intermetallics
}

\author{
Rasool Amini ${ }^{\mathrm{a}, *}$, Fatemeh Alijani ${ }^{\mathrm{a}}$, Mohammad Ghaffari ${ }^{\mathrm{b}}$, Morteza Alizadeh ${ }^{\mathrm{a}}$, Ali Kemal Okyay ${ }^{\mathrm{b}}$ \\ a Department of Materials Science and Engineering, Shiraz University of Technology, P.O. Box 71555-313, Shiraz, Iran \\ ${ }^{\mathrm{b}}$ Department of Electrical and Electronics Engineering, UNAM-Institute of Materials Science and Nanotechnology, Bilkent University, Ankara 06800, Turkey
}

\section{A R T I C L E I N F O}

Article history:

Received 12 July 2013

Received in revised form 29 October 2013

Accepted 14 December 2013

Available online 21 December 2013

\section{Keywords:}

Martensite

Austenite

Amorphization

Mechanical alloying

NiTi shape memory alloys

\begin{abstract}
A B S T R A C T
Ni-50Ti shape memory alloy was synthesized by mechanical alloying of the elemental powders mixture under an argon gas atmosphere. The structural and microstructural properties of the alloyed powders were evaluated by X-ray diffraction (XRD) and scanning electron microscope (SEM), respectively. Moreover, the Vickers microhardness of the powders was estimated at different milling times. According to the results, by milling progression, the amount of the amorphous phase increased considerably and after sufficient milling time ( $48 \mathrm{~h}$ ), the mechanocrystallization of the amorphous phase into the more stable crystalline phases (i.e. B2 and B19') occurred. It was found that the particles size and microhardness were significantly affected by the formation of the amorphous, B2, and B19' phases. It was also deduced that, by appropriate heating and cooling cycles, the B2 and thermally-induced B19' phases can be created. Furthermore, it was inferred that the formation of undesirable intermetallic phases (particularly $\mathrm{NiTi}_{2}$ ) during the heating cycle was considerably reduced by milling time evolution.
\end{abstract}

(C) 2013 Elsevier B.V. All rights reserved.

\section{Introduction}

NiTi shape memory alloy (SMA) exhibits remarkable properties such as shape memory effect (SME), superelasticity (SE) and biocompatibility [1]. SMAs are used in a wide range of medical [2], dental [3] and engineering applications [4-6]. NiTi (Nitinol) is an attractive biomaterial due to its biocompatible nature and excellent mechanical properties [1]. Although it was demonstrated that elementary nickel is a toxic agent and harmful to bone structures, NiTi is not genotoxic due to the ability of titanium to form a stable titanium oxide surface layer [2].

NiTi compound has two common crystalline structures: austenitic phase (B2) at high temperature with cubic structure and martensitic phase (B19') at low temperature with monoclinic structure. The transformation of these phases into each other creates the SME and SE due to thermal and mechanical transition, respectively [7].

NiTi SMA can be synthesized through different methods including liquid state [8-11] and solid state [12-19] routes. Among solid state techniques, mechanical alloying (MA) is a less expensive and simple procedure to synthesize the supersaturated solid solutions, nanocrystals, and amorphous structures. During MA, powder particles are subjected to severe plastic deformation to produce alloyed powders at ambient temperature [20].

There is a wide literature on the mechano-synthesis and characterization of NiTi compounds [14-19]. However, to the best of our knowledge, the martensitic transformation during MA was not reported in

\footnotetext{
* Corresponding author. Tel.: +98 917811 1858; fax: +98 7117354520 .

E-mail address: ramini2002@gmail.com (R. Amini).
}

any work. In the present study, the production of B19' martensite and amorphous phases and their effects on the microhardness were evaluated during MA. Afterwards, the thermal stability of the aforementioned phases was investigated.

\section{Experimental procedure}

In this study, pure powders of titanium (>99\%) and nickel (>99.5\%) were mixed with 50:50 atomic ratio (55:45 wt.\%) and then mechanically alloyed in a planetary ball mill with tempered steel vial $(90 \mathrm{ml})$ and balls ( $5 * 20 \mathrm{~mm}$ and $7 * 10 \mathrm{~mm}$ ) under argon atmosphere. The milling was conducted at room temperature with speed and ball-to-powder mass ratio (BPR) of $450 \mathrm{rpm}$ and 20:1, respectively. It is well-known that in the ductile-ductile alloying systems, a small quantity of the powders becomes welded on to the surface of the container and grinding medium which prevents the excessive wear of the milling medium. Accordingly, in the present work, to reduce the amount of contamination, the powders obtained from the 3rd milling duration were used for the analysis.

In order to determine the amount of contamination during the milling process, an X-ray fluorescence analyzer (XRF, Philips PW2400) was used and then the quantitative values were extracted by the PAN analytical software. The structural properties and phase transformation of the powders during MA were evaluated by using powder X-ray diffraction (XRD, PAN analytical, X'pert Pro MPD) with the CuK $\alpha$ $(\lambda=0.154 \mathrm{~nm})$ radiation at $40 \mathrm{kV}$ and $40 \mathrm{~mA}$. The XRD data were collected at the step time of $3 \mathrm{~s}$ and step size $0.03^{\circ} \mathrm{s}$ in the $2 \theta$ range of 20 $80^{\circ}$. The qualitative analysis and Rietveld refinement were performed 
using Match (version 2.0.7) and MAUD (version 2.26) software packages, respectively. To estimate the amorphous phase fraction, the XRD profile of a mixture of $90 \mathrm{wt} . \%$ milled powders and $10 \mathrm{wt} . \%$ internal standard (fully crystalline Mo powders) was analyzed by Rietveld method and the amorphous phase quantity was calculated by the following equation [21].

$\mathrm{A}(\mathrm{wt} . \%)=\left(1-W_{S} / R_{S}\right) /\left(100-W_{S}\right) * 10^{4}$

where $W_{S}$ is the weight percentage of the internal standard added to the powders and $R_{S}$ is its concentration estimated by Rietveld refinement of the XRD results.

The particle size variation, interlayer thickness, and chemical composition homogeneity of the alloyed powders were studied by a scanning electron microscope (SEM, FEI, Nova Nanosem 430) coupled with energy dispersive X-ray spectroscopy (EDS). The mean powder particle size was estimated from SEM images of powder particles by image analyzer. The average size of about 20 particles was calculated and reported as mean powder particle size. Also, to evaluate the microstructural feature of the milled samples, the powder particles were dispersed in ethanol, dropped down to copper grid and characterized by a transmission electron microscope (TEM, FEI, Tecnai G2 F30).

A small amount of powder was compacted at 2 bar (200 Pa) pressure to small pellets with $4 \mathrm{~mm}$ in dia. followed by mounting for microhardness testing using a Vickers indenter at a $300 \mathrm{~g}$ load and $10 \mathrm{~s}$ dwell time. Before this measurement, the mounted powders were polished with grit sandpapers (1200P and 2000P). Vickers hardness was calculated as the applied load, $P$ (measured in $\mathrm{mN}$ ), over the surface area of the indentation, as measured by the long diagonal length of the indentation, $d$ (measured in $\mu \mathrm{m}$ ) [22]:

$H_{V}=1854.4 P / d^{2}$.

Since indentation results are often influenced by unavoidable statistical variations, each individual result in the present study is an average of 5 measurements.

To investigate the phase transitions occurring during annealing, a small amount of $24 \mathrm{~h}$ and $96 \mathrm{~h}$ milled powders were compacted into small pellets and encapsulated under vacuum condition in quartz tube and were heated up to $850{ }^{\circ} \mathrm{C}$ for $1 \mathrm{~h}$ and then water quenched.

\section{Results and discussion}

Fig. 1(a) depicts the XRD patterns of the powders milled for various times. As it can be seen, the peak sharpness of the initial materials (i.e. Ni and Ti) has decreased by increasing the milling time. By progression of milling, the peak broadening is increased due to introduction of high amount of energy and defects. By increasing the milling time, Ti peaks disappeared faster than Ni peaks and are completely eliminated after $12 \mathrm{~h}$ of milling. Further analysis of the XRD spectra of the initial milling durations indicates that the diffraction lines of Ni shift toward lower angles and the lattice parameter of Ni increases (Fig. 1(b)) owing to the dissolution of Ti in Ni lattice and solid solution formation. Due to applying a high energy to the powders and continuous reduction of the effective crystallite size [20], the amorphous phase has formed at the initial milling times and grown considerably by progression of milling up to $24 \mathrm{~h}$. More focus on the broadening of the $24 \mathrm{~h}$-milled powders revealed that the amorphization process has occurred at this milling interval. At this time the maximum amount of amorphous phase was achieved.

It is clear that severe plastic deformation introduced into the powders during MA creates a variety of crystalline defects such as dislocations, stacking faults and grain boundaries $[20,23]$. Since the presence of the defects decreases the diffusion distances, the diffusivity of Ti as a solute element into Ni enhances and with the slight rise in the milling temperature, true alloying takes place among the constituent elements. Comparing the XRD patterns of the powders milled for $24 \mathrm{~h}$ and $96 \mathrm{~h}$
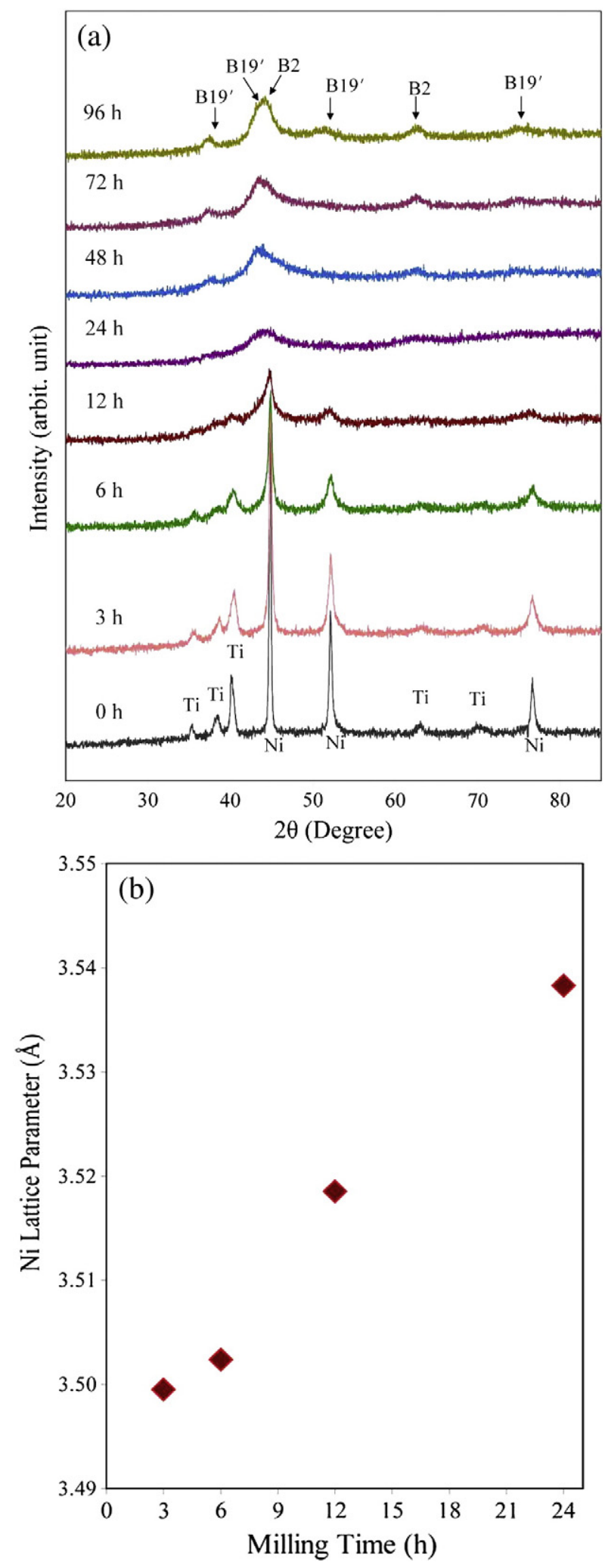

Fig. 1. (a) The XRD pattern of NiTi compound at various milling times and (b) the variation of Ni lattice parameter as a function of milling time.

indicates that the mechanical crystallization of the amorphous phase to the more stable crystalline B2-austenite and B19'-martensite phases occurs during the milling interval and consequently the amount of the amorphous phase is decreased. This event attributed to strain energy and a temperature increase during MA [20] which has been previously observed in Ni-Ti-Ta system [24]. 
Table 1

Quantitative XRD results of the milled powders.

\begin{tabular}{|c|c|c|c|c|c|c|c|c|c|c|c|c|c|}
\hline \multirow[t]{2}{*}{ Milling Time (h) } & \multicolumn{3}{|l|}{$\mathrm{Ni}$} & \multicolumn{3}{|l|}{$\mathrm{Ti}$} & \multicolumn{3}{|c|}{ B19' } & \multicolumn{3}{|l|}{ B2 } & \multirow{2}{*}{$\frac{\text { Amorphous }}{\text { wt.\% }}$} \\
\hline & $\mathrm{D}$ & $\varepsilon$ & wt.\% & $\mathrm{D}$ & $\varepsilon$ & wt.\% & $\mathrm{D}$ & $\varepsilon$ & wt.\% & $\mathrm{D}$ & $\varepsilon$ & wt.\% & \\
\hline 3 & 44 & 0.120 & 75 & 42 & 0.533 & 15 & & & & & & & 10 \\
\hline 6 & 27 & 0.254 & 54 & 22 & 0.726 & 10 & & & & & & & 36 \\
\hline 12 & 15 & 0.557 & 42 & 12 & 0.912 & 8 & 78 & 0.396 & 5 & 89 & 0.200 & 2 & 43 \\
\hline 24 & II & 0.695 & 5 & & & & 46 & 0.529 & 19 & 52 & 0.352 & 6 & 70 \\
\hline 48 & & & & & & & 32 & 0.768 & 23 & 31 & 0.567 & 16 & 61 \\
\hline 72 & & & & & & & 29 & 0.893 & 20 & 22 & 0.710 & 25 & 55 \\
\hline 96 & & & & & & & 18 & 1.010 & 34 & 16 & 0.869 & 18 & 48 \\
\hline
\end{tabular}

D: Crystallite size (nm). $\varepsilon$ : r.m.s. microstrain (\%). wt.\%: Weight percent.

The variation of phase content as a function of milling time is listed in Table 1 . As can be seen, by starting the milling process, the quantity of elemental powders decreases and the amount of $\mathrm{Ni}$ solid solution and amorphous phase increase; moreover, the formation of B19' martensite and B2 austenite phases are indicated. By increasing the milling duration, not only the stress-induced martensite (B19') is formed due to severe plastic deformation, but also the high temperature B2 phase is also created due to temperature rising. By progression of milling up to $24 \mathrm{~h}$, the fraction of the amorphous phase increased and then due to mechano-crystallization of the phase into more stable crystalline phases (B19' and B2), its amount is reduced. According to the quantitativeresults extracted from XRD test, the B19' and B2 phases, at initial milling stages (up to $24 \mathrm{~h}$ ), are produced directly from the primary materials and afterwards from the amorphous phase after mechanocrystallization process.

Fig. 2 shows HRTEM image and the corresponding SAD pattern of the $96 \mathrm{~h}$ milled powders. According to the image (Fig. 2(a)), the average crystallite size of the crystalline phases reaches to value of about $15 \mathrm{~nm}$ at the end of the process (i.e. $96 \mathrm{~h}$ ). As it can be seen from the SAD pattern (Fig. 2(b)), the coexistence of amorphous and nanocrystalline phases (B19' and B2) in the alloyed powders is confirmed respectively by the halo appearance and continuous rings which is compatible with the aforementioned XRD results.

Figs. 3 and 4 include the SEM images and the analyzed average particles size of the milled powders at different milling times. As shown in the figures, $1 \mathrm{~h}$ milled powder contains fine particles with irregular shapes in which by milling evolution up to $6 \mathrm{~h}$, due to high surface energy of fine and ductile particles, a maximum cold welding and agglomeration occurs and particles size increases considerably and a broad of particle size distribution is achieved. At this milling interval, the composite lamellar structure with layer thicknesses of about $1 \mu \mathrm{m}$ was formed. By progression of milling (i.e. $24 \mathrm{~h}$ ), due to domination of the fracturing over cold welding and increase in the amount of amorphous phase, the brittleness of the powders increases; consequently, the particle size is significantly reduced and their morphology tends to be semispherical with a narrow range of size distribution (Fig. 4). Afterwards, a balance between the rates of cold welding and fracturing is achieved and the particles size reaches its steady state condition [23,25], which is associated with the narrow particles size distribution and saturation in hardness [25]. At this stage, the composite lamellar structure diminishes and therefore the alloying process is completed. By milling evolution to $48 \mathrm{~h}$, due to occurrence of the mechano-crystallization of the amorphous phase to the more stable B19'martensite and B2 austenite phases, the powders ductility is enhanced and subsequently the particles size increases significantly. At the final stages of milling, because of the development of hard B2 phase, the fracturing process overcomes the cold welding phenomenon and consequently the particle size refinement occurred once more.

Fig. 5 depicts the EDS elemental mapping and point spectrum of the $96 \mathrm{~h}$-milled powders, wherein the uniform elements distribution is obvious. However, a small quantity of iron impurity is detectable in the point spectrum. It has been reported that the Fe addition to binary Ti$\mathrm{Ni}$ alloys is very effective to separate the pre-martensitic and martensitic transformation temperature ranges [26]. Moreover, the martensitic transformation start temperature (Ms) is significantly reduced by substitution of a considerable amount of Fe (e.g. 2.6 wt.\%)with $\mathrm{Ni}$ in $\mathrm{Ti}-\mathrm{Ni}$ alloys. In order to determine the exact amount of iron in the alloyed powders, the XRF test was also performed on the powders. According to the results, the iron content of the samples milled for $96 \mathrm{~h}$ was about $0.8 \mathrm{wt}$.\% which is fairly low, revealing that the milling conditions are suitable for the MA process of the present alloying system.

Fig. 6 shows the average microhardness of the prepared samples at various milling times. As it can be seen, by starting the MA process, the samples microhardness is increased due to the introduction of
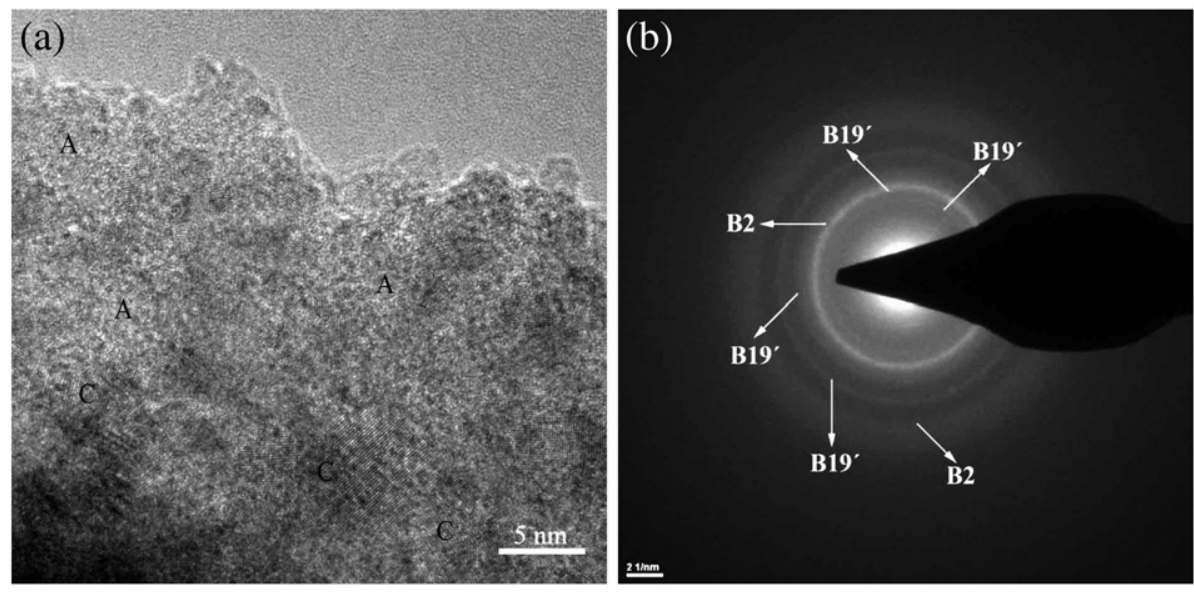

Fig. 2. (a) HRTEM image of 96 h-milled powders indicated the amorphous, A, and crystalline, C, parts of the structure and (b) the corresponding SAD pattern. 

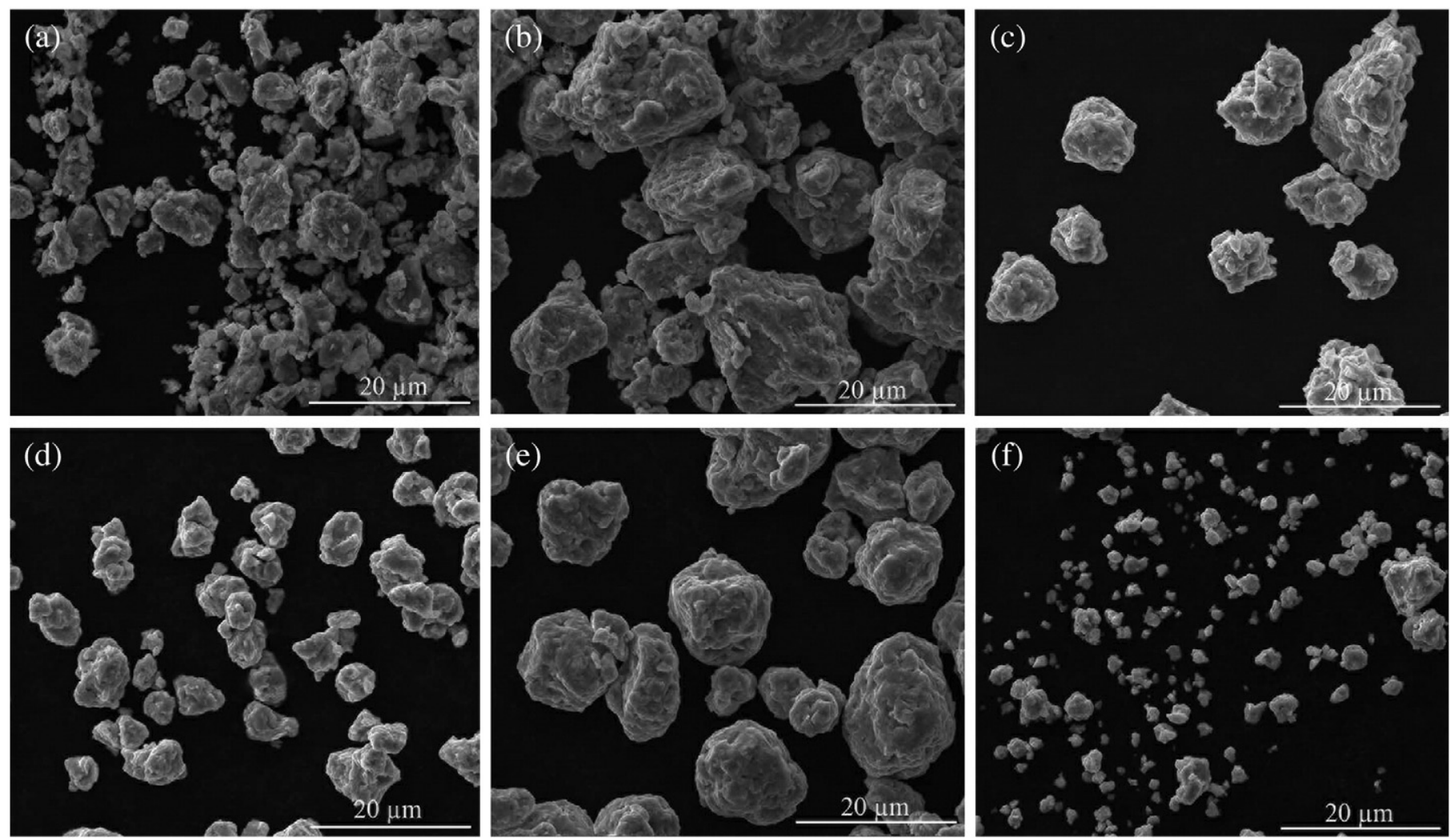

Fig. 3. The SEM images of the (a) 1 h, (b) 6 h, (c) 12 h, (d) 24 h, (e) 48 h, and (f) 96 h milled powders.

high density of defects into the powders structure and continuous enhancement of the amorphous phase quantity. At $24 \mathrm{~h}$ of milling, due to the presence of high fraction of amorphous phase and hard B2-NiTi phase, the Vickers hardness reaches its maximum value which is higher than the values reported in the literatures [27-29]. By further milling (up to $48 \mathrm{~h}$ ), the samples microhardness is drastically reduced which is attributed to the mechano-crystallization of the amorphous phase and enhancement of soft B19' phase at 24 h-to-48 h durations. At the end of process (i.e. $72 \mathrm{~h}$ and $96 \mathrm{~h}$ ), by increasing the amount of hard B2 phase, the microhardness of the alloyed samples is re-increased.

In order to evaluate the formation probability of thermally induced martensite, the selected mechanically alloyed samples ( $24 \mathrm{~h}$ and $96 \mathrm{~h}$ ) were heated up to the temperatures well above the crystallization temperature of the amorphous phase (at which the B2-austenite phase is stable (e.g. $\left.850{ }^{\circ} \mathrm{C}\right)$ ) and remained for $1 \mathrm{~h}$ and then water quenched. Afterwards, the XRD analysis was conducted on the samples. Concerning

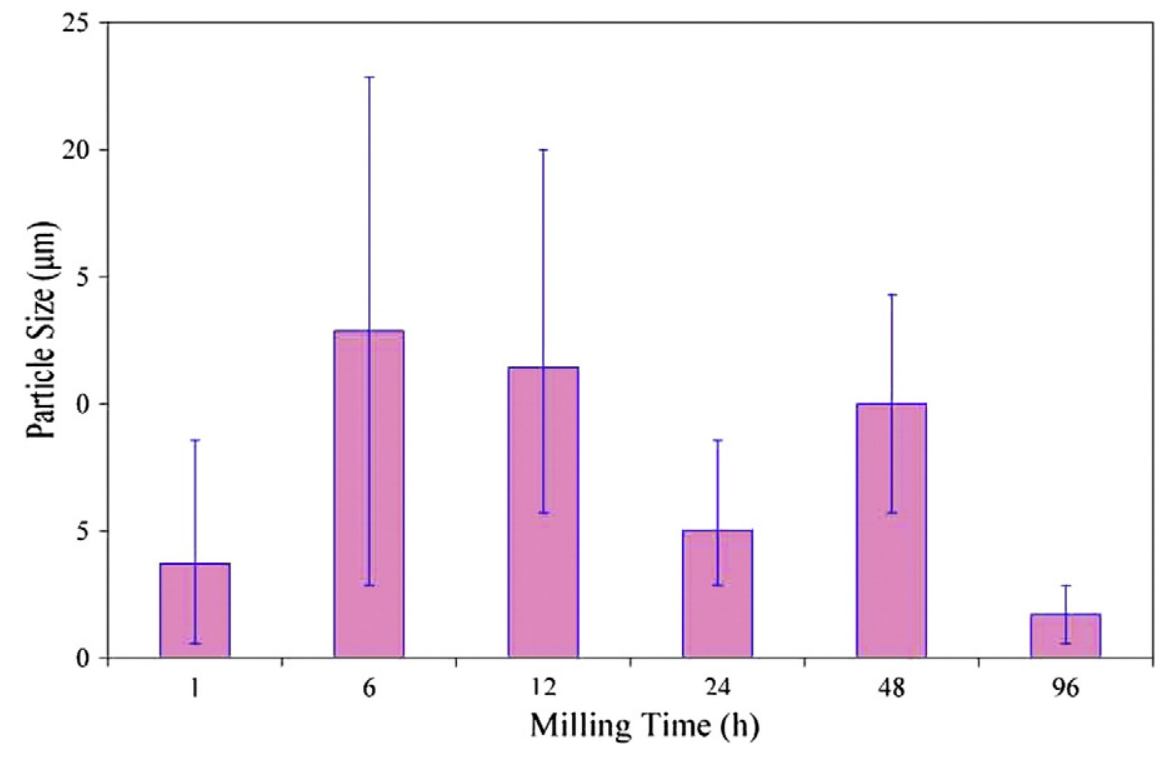

Fig. 4. The variation of particle size by milling time. 

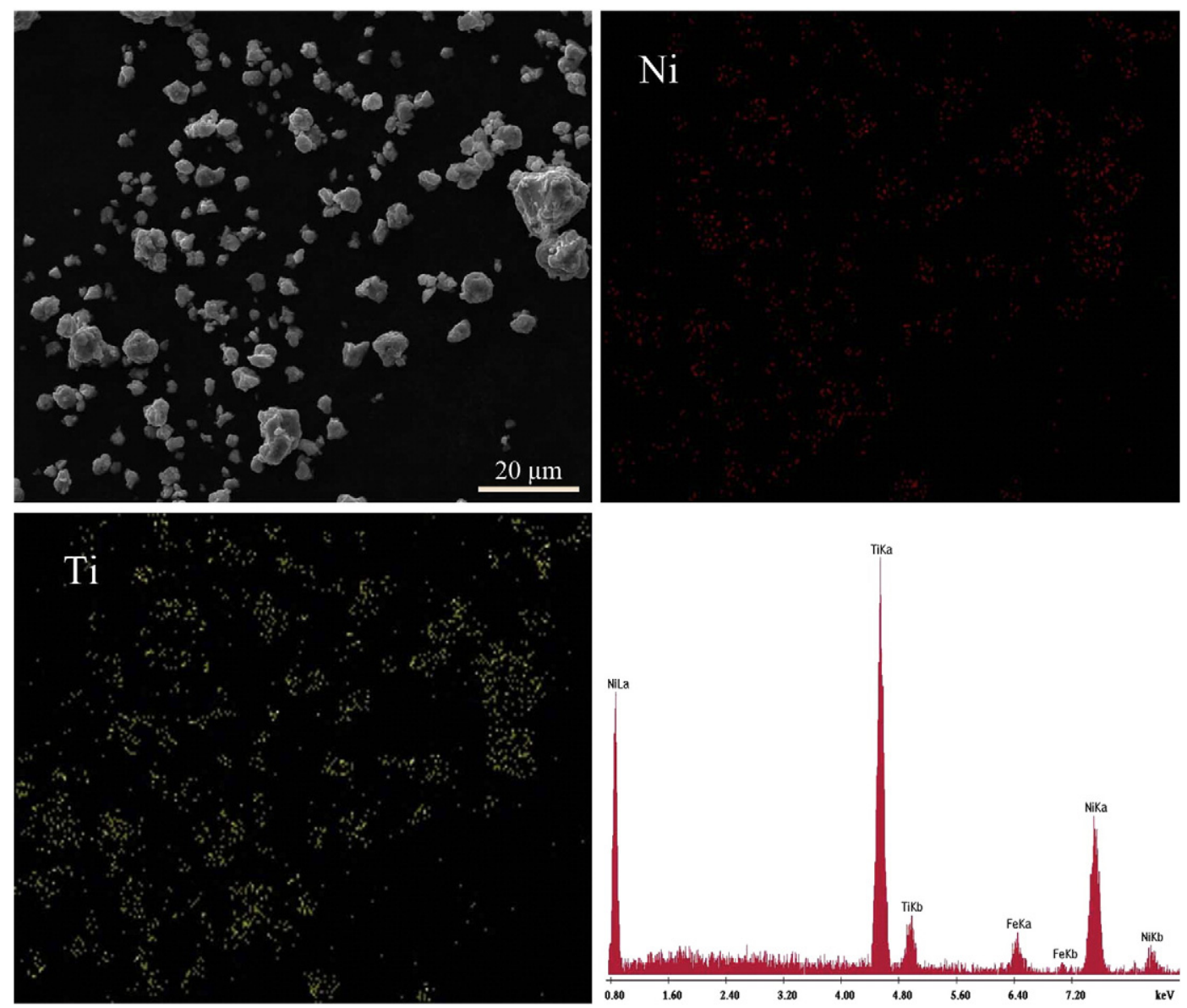

Fig. 5. Element distribution in the $96 \mathrm{~h}$ milled powders.

Fig. 6, it is evident that thermally-assisted martensite with B19' structure is the major phase in which its amount is significantly high in the $96 \mathrm{~h}$ milled sample with respect to the sample milled for $24 \mathrm{~h}$. That is, the transformation temperature of B2-to-B19' $\left(\mathrm{M}_{\mathrm{S}}\right)$ is above the room temperature wherein B2-NiTi can be partially transformed into B19'-NiTi after the cooling cycle. More focus on the XRD patterns and the correlated table of Fig. 7 reveals that in addition to the B19' and B2 phases, a significant quantity of undesirable $\mathrm{Ni}_{3} \mathrm{Ti}$ and $\mathrm{NiTi}_{2}$ are formed in the sample milled for $24 \mathrm{~h}$; while, in the $96 \mathrm{~h}$ milled sample, the amount of $\mathrm{Ni}_{3} \mathrm{Ti}$ phase decreases considerably and $\mathrm{NiTi}_{2}$ is completely vanished. It can be attributed to the increase in the homogeneity of the amorphous phase by milling development.

\section{Conclusions}

In the present work, the amorphous/nanocrystalline Ni-50Ti alloy was successfully produced by mechanical alloying of the elemental $\mathrm{Ti}$ and Ni powders and their structure, microstructure, and microhardness were investigated. Accordingly, the most important results are summarized as follows:

1) At the initial stages of milling, the elemental powders dissolved into the structure in which the Ti peaks diminished faster than Ni peaks (i.e. $12 \mathrm{~h}$ ) and the supersaturated solid solution of $\mathrm{Ti}$ in $\mathrm{Ni}$ was formed.

2) By milling progress, the amorphization process occurred and the amorphous phase fraction was increased reaching its maximum value (70 wt.\%) at $24 \mathrm{~h}$ of milling. Simultaneously, the average particles size was reduced and microhardness was increased significantly reaching its greatest value of about $522 \mathrm{Hv}$ after $24 \mathrm{~h}$ of milling.

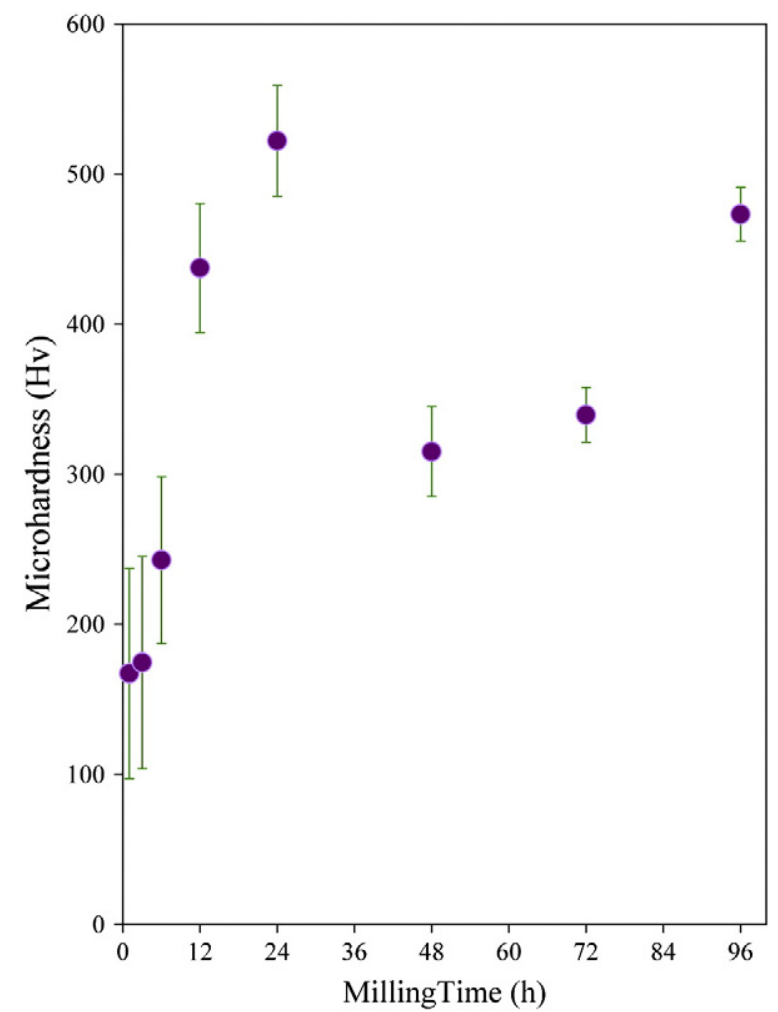

Fig. 6. The variation of the Vickers microhardness of the prepared pellets as a function of milling time. 


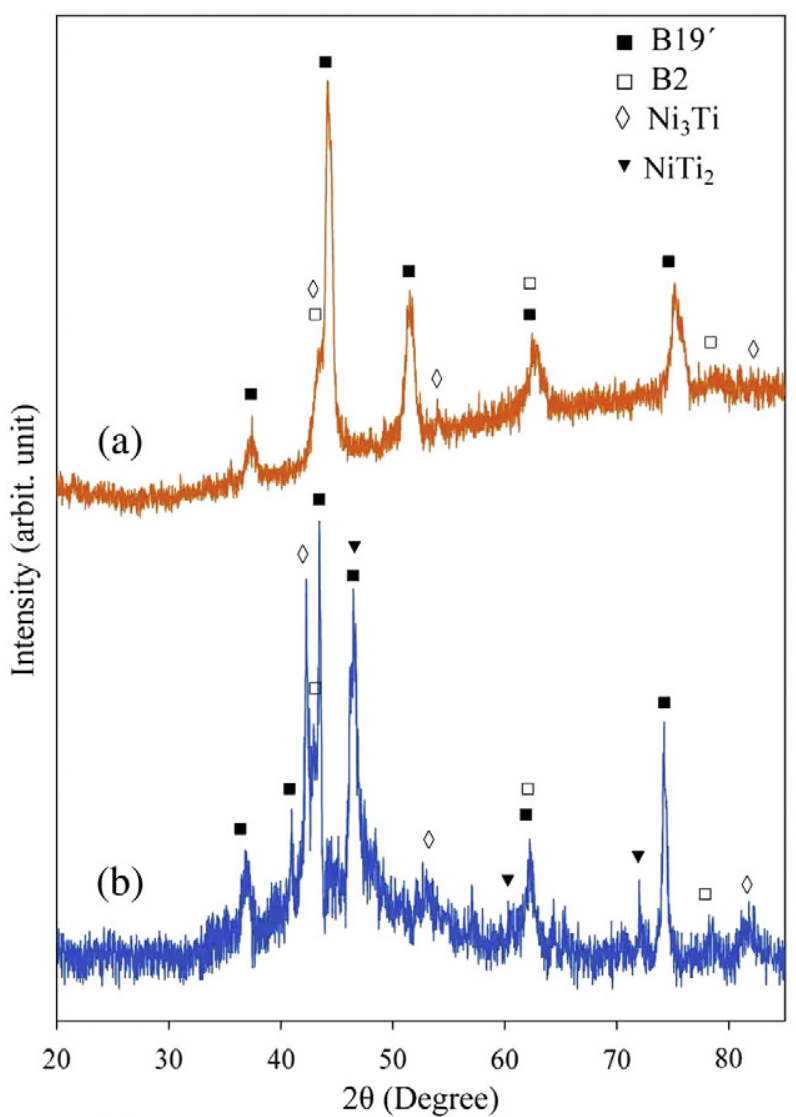

(c)

\begin{tabular}{|c|c|c|c|c|}
\hline Milling Time (h) & $\overline{\mathrm{B} 19^{\prime}}$ & B2 & $\mathrm{Ni}_{3} \mathrm{Ti}$ & $\mathrm{NiTi}_{2}$ \\
\hline 24 & $46(3)$ & $14(2)$ & $35(3)$ & $5(1)$ \\
\hline 96 & $73(5)$ & $9(1)$ & $17(2)$ & - \\
\hline
\end{tabular}

Error value $(\mathrm{X}): \pm \mathrm{X}$ wt.\%.

Fig. 7. The XRD pattern of NiTi alloy milled for (a) $96 \mathrm{~h}$ and (b) $24 \mathrm{~h}$ followed by heat treatment at $850{ }^{\circ} \mathrm{C}$ for $1 \mathrm{~h}$. (c) The variation of phase content of the samples by milling time.

3) After sufficient milling time (i. e. $48 \mathrm{~h}$ ), the mechano-crystallization of the amorphous phase into the more stable crystalline phases containing nanocrystalline B19' and B2 occurred and the fraction of the amorphous phase was reduced. Concurrently, the average particle size was increased and the powders microhardness was reduced extensively.

4) At final stages of milling, the hard B2 austenite phase was developed considerably and as a consequence the average particles size was reduced and microhardness was increased once again.

5) In the present alloying system, the transformation temperature of B2-to-B19' $\left(\mathrm{M}_{\mathrm{S}}\right)$ is higher than room temperature and consequently, the production of thermally induced B19'-martensite phase is feasible.
6) The formation of undesirable intermetallic phases (e.g. $\mathrm{Ni}_{3} \mathrm{Ti}$ and $\mathrm{NiTi}_{2}$ ) during the heating cycle was significantly impeded by milling evolution (up to $96 \mathrm{~h}$ ).

\section{References}

[1] D.C. Lagoudas, Shape Memory Alloys Modeling and Engineering Applications, Springer, Texas, 2008.

[2] A.R. Pelton, D. Stöckel, T.W. Duerig, Medical uses of nitinol, Mater. Sci. Forum 63 (2000) 327-328.

[3] S.A. Thompson, An overview of nickel-titanium alloys used in dentistry, Int. Endod. J. 33 (2000) 297-310.

[4] J.V. Humbeeck, Non-medical applications of shape memory alloys, Mater. Sci. Eng. A 134 (1999) 273-275.

[5] W. Predki, A. Knopik, B. Bauer, Engineering applications of NiTi shape memory alloys, Mater. Sci. Eng. A 598 (2008) 481-482.

[6] W. Huang, On the selection of shape memory alloys for actuators, Mater. Des. 23 (2002) 11-19.

[7] K. Otsuka, X. Ren, Recent developments in the research of shape memory alloys, Intermetallics 7 (1999) 511-528.

[8] H. Matsumoto, Addition of an element to NiTi alloy by an electron-beam melting method, J. Mater. Sci. Lett. 10 (1991) 417-419.

[9] G.M. Kramer, A comparison of chemistry and inclusion distribution and morphology versus melting method of NiTi alloys, J. Mater. Eng. Perform. 18 (2009) 479-483.

[10] L. Zhang, C. Xie, J. Wu, Martensitic transformation and shape memory effect of Ti49 at.\%Ni alloys, Mater. Sci. Eng. A 438-440 (2006) 905-910.

[11] J.I. Kim, S. Miyazaki, Effect of nano-scaled precipitates on shape memory behavior of Ti-50.9 at.\%Ni alloy, Acta Mater. 53 (2005) 4545-4554.

[12] B. Li, L. Rong, Y. Li, Stress-strain behavior of porous Ni-Ti shape memory intermetallics synthesized from powder sintering, Intermetallics 8 (2000) 643-646.

[13] S. Tria, O. Elkedim, R. Hamzaoui, X. Guo, F. Bernard, N. Millot, O. Rapaud, Deposition and characterization of cold sprayed nanocrystalline NiTi, Powder Technol. 210 (2011) 181-188

[14] T. Nam, S. Kang, Effect of ball milling conditions on the microstructure and the transformation behavior of $\mathrm{Ti}-\mathrm{Ni}$ and $\mathrm{Ti}-\mathrm{Ni}$-Cu shape memory alloy powders, Met Mater. Int. 8 (2002) 145-150.

[15] Y.W. Gu, C.W. Goh, L.S. Goi, C.S. Lim, A.E.W. Jarfors, B.Y. Tay, M.S. Yong, Solid state synthesis of nanocrystalline and/or amorphous 50Ni-50Ti alloy, Mater. Sci. Eng. A 392 (2005) 222-228.

[16] D. Sun, L. Cheng, Y. Zhang, K. Ho, Calorimetry study of $\mathrm{Ni}_{50} \mathrm{Ti}_{50}$ ball milled powders, J. Alloys Compd. 186 (1992) 33-35.

[17] R.B. Schwarz, R.R. Petrich, C.K. Saw, The synthesis of amorphous Ni-Ti alloy powders by mechanical alloying, J. Non-Cryst. Solids 76 (1985) 281-302.

[18] D.D. Radev, Mechanical synthesis of nanostructured titanium-nickel alloys, Adv. Powder Technol. 21 (2010) 477-482.

[19] W. Maziarz, J. Dutkiewicz, J. Van Humbeeck, T. Czeppe, Mechanically alloyed and hot pressed Ni-49.7Ti alloy showing martensitic transformation, Mater. Sci. Eng. A 375-377 (2004) 844-848

[20] C.C. Koch, J.D. Whittenberge, Mechanical milling/alloying of intermetallics, Intermetallics 4 (1996) 339-355.

[21] R. Amini, M.J. Hadianfard, E. Salahinejad, M. Marasi, T. Sritharan, Microstructural phase evaluation of high-nitrogen $\mathrm{Fe}-\mathrm{Cr}$-Mn alloy powders synthesized by the mechanical alloying process, J. Mater. Sci. 44 (2009) 136-148.

[22] ASTM Designation, Standard Test Method for Microindentation Hardness of Materials, ASTM, Philadelphia, 1999. 384-399.

[23] C. Suryanarayana, T. Klassen, E. Ivanov, Synthesis of nanocomposites and amorphous alloys by mechanical alloying. J. Mater. Sci. 46 (2011) 6301-6315.

[24] S. Kanchibhotla, N. Munroe, T. Kartikeyan, Amorphization in Ni-Ti-Ta system through mechanical alloying, J. Mater. Sci. 40 (2005) 5003-5006.

[25] J.S. Benjamin, T.E. Volin, The mechanism of mechanical alloying, Metall. Trans. 5 (1974) 1929-1934.

[26] M. Nishida, C.M. Wayman, T. Honma, Phase transformation in Ti50Ni47.5Fe2.5 shape memory alloy, Metallography 19 (1986) 99-113.

[27] D.E. Burkes, G. Gottoli, H.C. Yi, J.J. Moore, Combustion synthesis and mechanical properties of dense NiTi-TiC intermetallic-ceramic composites, Metall. Mater. Trans. A 37A (2006) 235-242.

[28] K. Gall, K. Juntunen, H.J. Maier, H. Sehitoglu, Y.I. Chumlyakov, Instrumented micro-indentation of NiTi shape memory alloys, Acta Mater. 49 (2001) 3205-3217.

[29] K.Y. Chiu, F.T. Cheng, H.C. Man, Laser cladding of austenitic stainless steel using NiTi strips for resisting cavitation erosion, Mater. Sci. Eng. A 402 (2005) 126-134. 